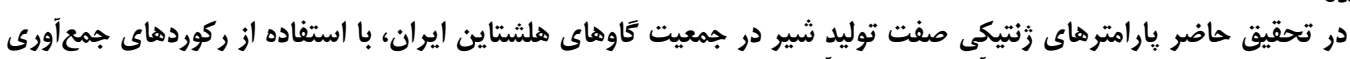

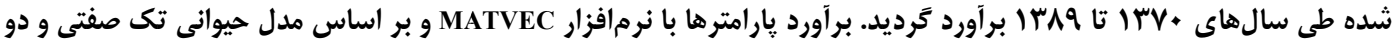

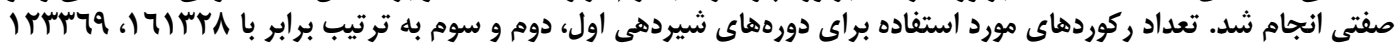

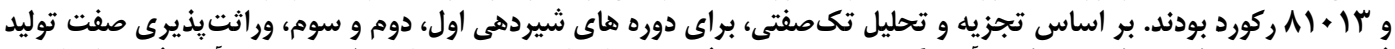

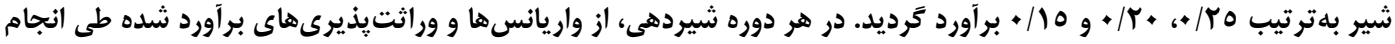

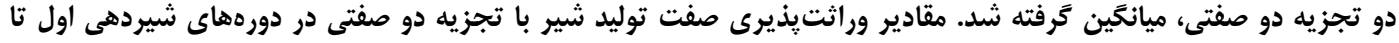

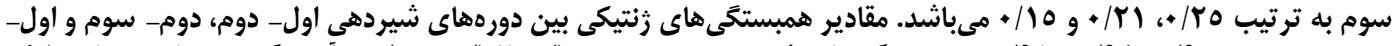

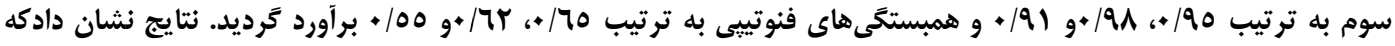

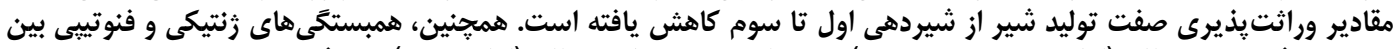

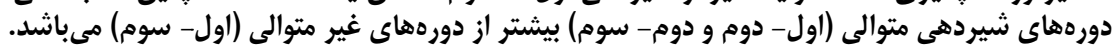

وازههاى كليدى: بارامترهاى زنتيكى، تجزيه تك صفتى، تجزيه دو صفتى، شيردهى

اولين دوره شيردهى يا از تجزيه و تحليل ركوردهاى تكرار

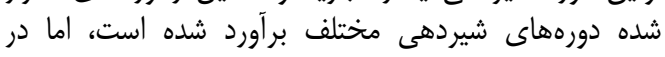

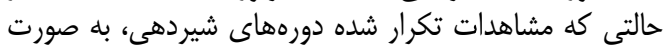

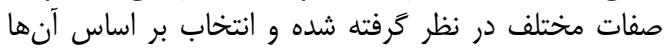

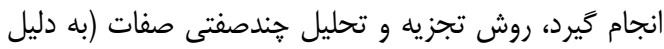

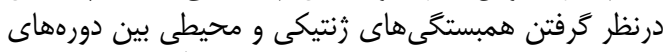

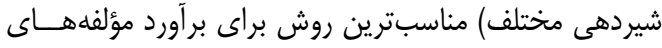

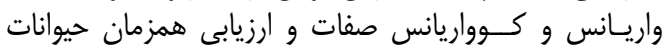

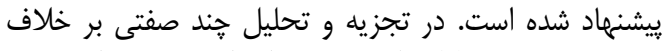

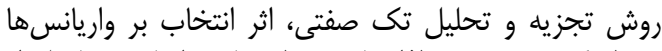

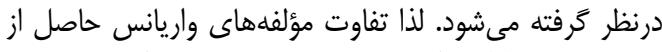
تجزيه و تحليل تكى و و

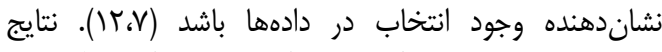

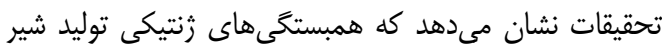

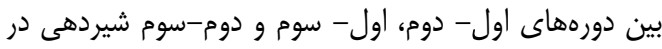

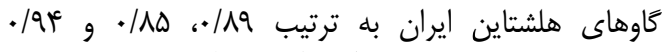

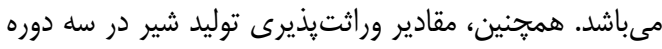

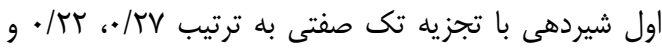

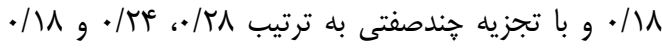

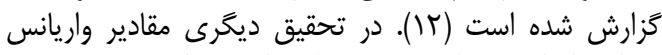

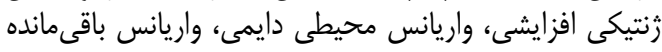

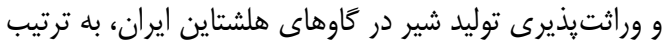

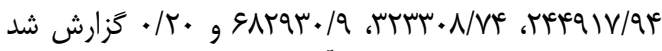

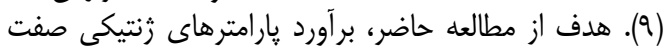

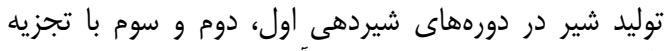
تك صفتى و دو صفتى و مقايسه آنها مى دياشد.

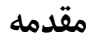

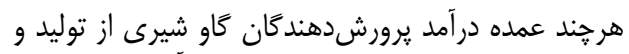

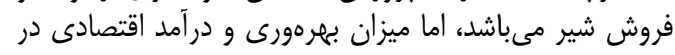

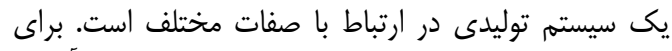

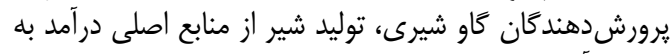

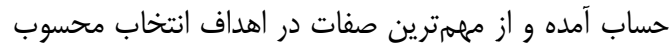

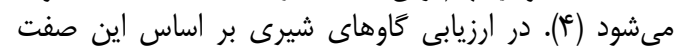

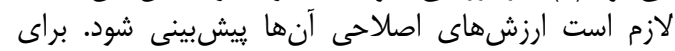

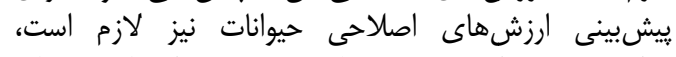

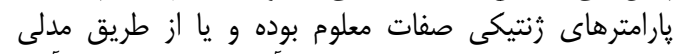

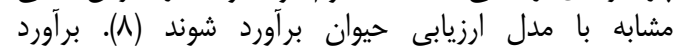

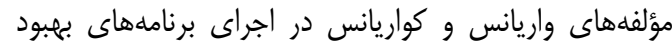

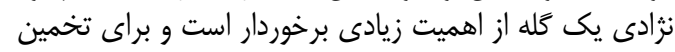

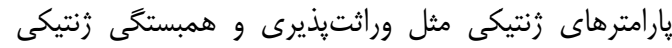

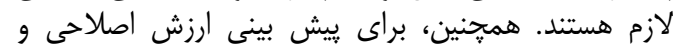

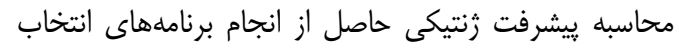

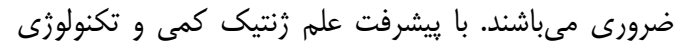

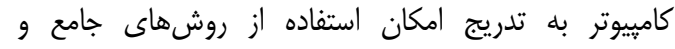

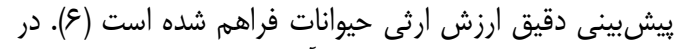

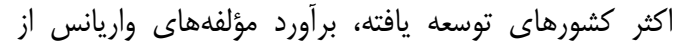

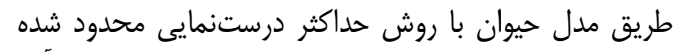

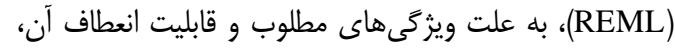

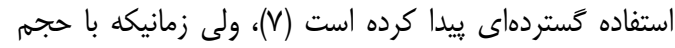

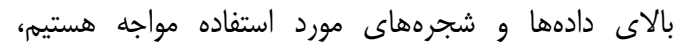

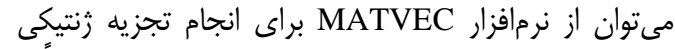

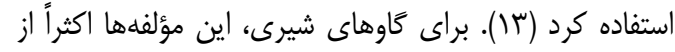

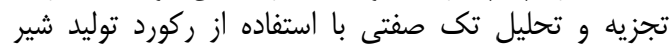




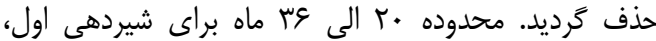

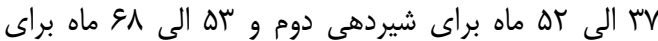

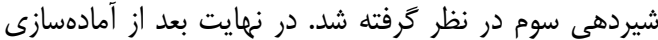

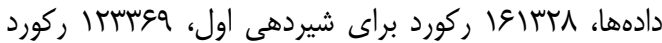

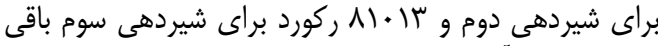

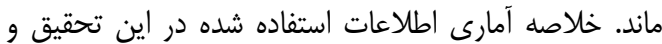
اطلاعات فايل شجره در جداول الو ب نشان داده شده است.

\section{مواد و و روشها}

الف) دادهها

در بررسى حاضر از دادههاى صفت توليد شادها شير، سه دوره إنها

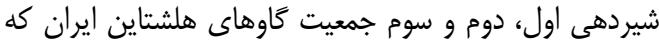

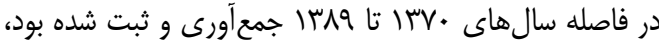

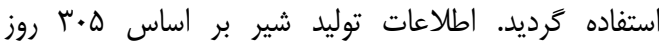

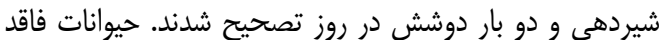

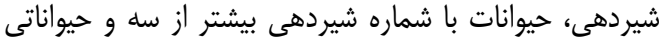

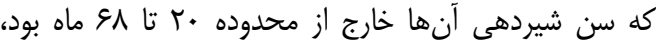

Table 1. Statistical summary for milk production data in first three lactation

جدول ا- خلاصه آمارى دادههاى توليد شير زايش اول، دوم و سوم

\begin{tabular}{|c|c|c|c|c|c|c|}
\hline ضريب جولغى & ضريب تغييرات & اشتباه معيار & انحراف معيار & ميانغين & تعداد & \\
\hline$-\cdot /|r|$ & $\overline{T T / R q}$ & $r / \kappa \omega$ & ITAV/AV & $9 T \cdot F / T \Lambda$ & 19ITYN & شيردهى اول \\
\hline.$- / .1$ & r ג . & $F / Q q$ & $|\varnothing| \Delta / \cdot V$ & $S V A F / \Delta V$ & 99 & شيردهى دوم \\
\hline.$- / .4 \Delta$ & $r \varphi / . r$ & D/AY & $1999 / 1 \Lambda$ & $V \cdot F \cdot / F \Delta$ & און & شيردهى سوم \\
\hline
\end{tabular}

Table 2. Pedigree information for whole milk production data

جدول r- اطلاعات شجرهاى كل دادهاى توليد شير

\begin{tabular}{|c|c|c|c|c|c|}
\hline حيوانات بدون فرزند & حيوانات داراى فرزند & 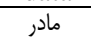 & 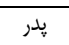 & تعداد حيوان همخون & كل حيوانات موجود در فايل شجره \\
\hline $1 K r \cdot 19$ & IFTIEY & IrGAFT & DGY. & 1. rDq. & TEFIAT \\
\hline
\end{tabular}

روردهاى مربوط به توليد شير در شيردهى ساى

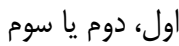

ماتريس طرح دوم عوامل ثابت براى شيردهىهاى ماول، دوم يا سوم ماتر سرح

ماتريس طرح عوامل تصادفى سوم براى شيردهى دهاى اول، دوم يا سوم طرح عاتر

بردار عوامل ثابت براى صفات شيردهى اول، دوم يا سوم

بردار عوامل تصادفى براى صفات شيردهى اول، دوم يا سوم

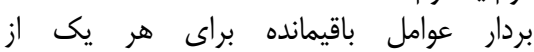
شيردهى هاى اول، دوم يا سوم.

$\mathrm{y} 2, \mathrm{y} 1$

$\mathrm{x} 2, \mathrm{x} 1$

$\mathrm{z} 2, \mathrm{z} 1$

b2, b1

$\mathrm{u} 2, \mathrm{u} 1$

e2, e1

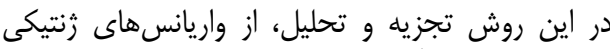

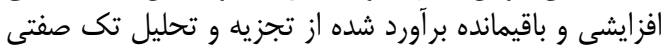

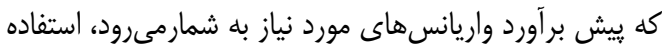

تجزيه و تحليل دادهها با استفاده از نرمافزار و از طريق مدل حيوانى صورت تحرفت. تحها

\section{نتايج و بحث}

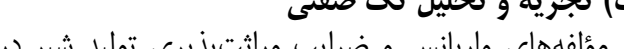

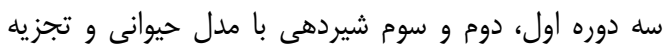
تكى صفتى در جدول سو ارايه شده است.
ب) تجزيه و تحليل آمارى تجلي تجزيه و تحليل و تحليل امارى صفتى: در تجزيه تكى صفتى

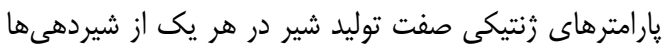

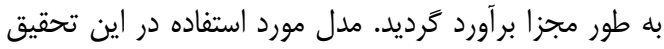
به شرح زير مى باشد: $Y_{i j k l}=\mu+H_{i}+A_{j e}+a_{k}+e_{i j k l}$

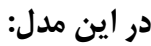
= = مشاهده lijk

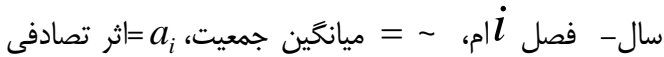
k امين حيوان، HYS فصل، Ag= اثر سن در زايش ز ز ام ( = = اثر باقيمانده. تجزيه و تحليل دو صفتى:

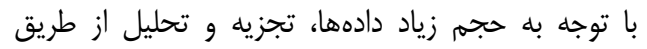

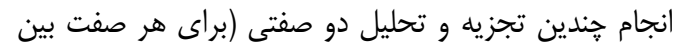

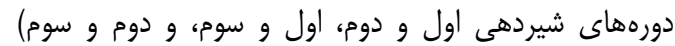

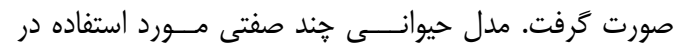
اين تحقيق بلصورت زئ مدر حيوان باشد:

$\left[\begin{array}{ll}x_{1} & 0 \\ 0 & x_{2}\end{array}\right]+\left[\begin{array}{l}a_{1} \\ a_{2}\end{array}\right]\left[\begin{array}{ll}z_{1} & 0 \\ 0 & z_{2}\end{array}\right]+\left[\begin{array}{l}e_{1} \\ e_{2}\end{array}\right]$ $\left[\begin{array}{l}y_{1} \\ y_{2}\end{array}\right]=\left[\begin{array}{l}b_{1} \\ b_{2}\end{array}\right]$ 
جدول س- مؤلفههاى واريانس و وراثتيذيرى صفت توليد شير درسه دوره اول شيردهى در جمعيت گاوهاى هلشتاين ايران Table 3. Variance components and heritability for first three lactation in Iranian Holstein dairy cattle

\begin{tabular}{|c|c|c|c|c|}
\hline$h_{(\mathrm{SE})}^{2}$ & $\sigma_{p}^{2}$ & $\sigma_{e}^{2}$ & $\sigma_{a}^{2}$ & \\
\hline$\cdot / T \Delta_{(\cdot / \cdot 1)}$ & IIDTGAF & NEFFET & TAQT. & شيردهى اول \\
\hline$\cdot / r \cdot(\cdot / 1)$ & IFIfIVT & ITADIV. & rrq..r & شيردهى دوم \\
\hline$\cdot / 1 \Delta_{(\cdot / \cdot r)}$ & $\left|A V^{\prime}\right| g V$ & lo9Ver. & TVAVTrY & شيردهى سوم \\
\hline
\end{tabular}

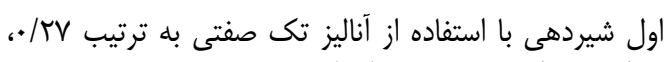

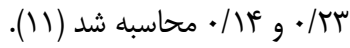
ب) تجزيه و تحليل دو صفتى

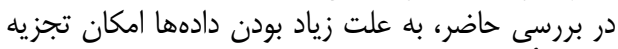

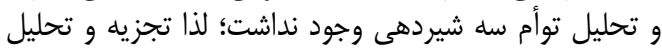

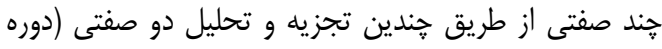

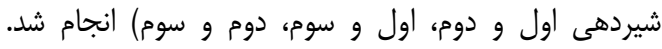

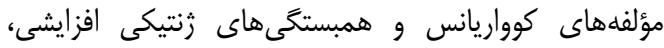

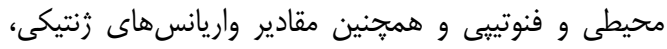

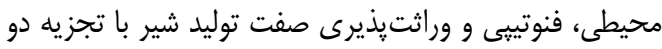

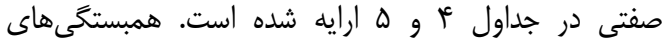

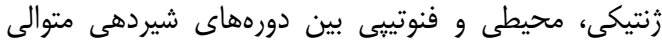

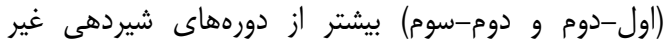

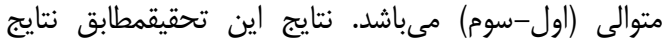
تحقيق كَارسيا- كورتز و همكاران (ه) مى مباشد.
نتايج نشان داد كه، مقادير وراثتيذيرى صفت توليد شير

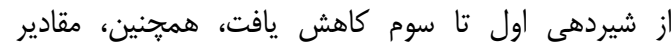

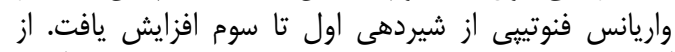

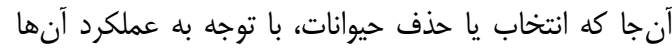

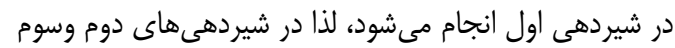

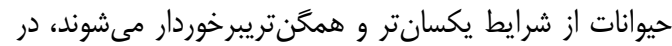

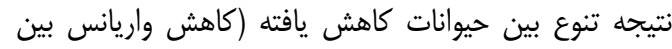

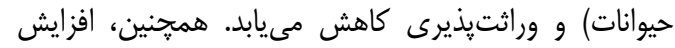

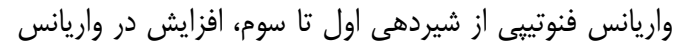

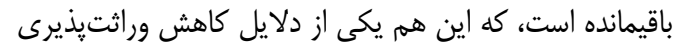

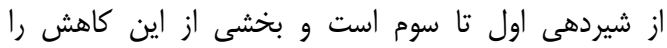

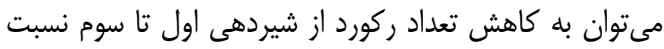

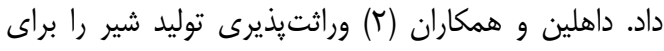

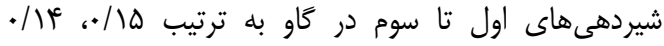

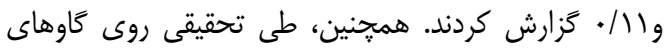

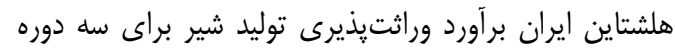

جدول أ- مؤلفهاى كوواريانس و همبستخى هاى زنتيكى افزايشى، محيطى و فنوتييى صفت توليد شير در سه دوره شيردهى Table 4. Variance components and genetic, phenotypic and environmental correlations in first three lactation

\begin{tabular}{|c|c|c|c|c|c|c|c|}
\hline$r_{p i j}$ & $r_{e i j}$ & $r_{a i j}$ & $\sigma_{p i j}$ & $\sigma_{e i j}$ & $\sigma_{a i j}$ & صفت دوم (شيردهى) & صفت اول (شيردهى) \\
\hline.$/ 90$ & $\cdot / \Delta V$ &.$/ 90$ & 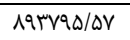 & $G \cdot V \Delta Q / \Delta V$ & rq५.rq & دوم & اول \\
\hline$\cdot / \Delta \Delta$ & $\cdot / \uparrow \Lambda$ & $\cdot / 91$ & $N r \cdot 99 \cdot / r r$ & $\Delta G F \cdot M F / T Y$ & rDSQDS & سوم & اول \\
\hline . Mt & $\cdot / \Delta F$ &.$/ 91$ & $1.97 \times 99 / . r$ & N.r req/.r & TQD।Q. & سوم سوم & دوم \\
\hline
\end{tabular}

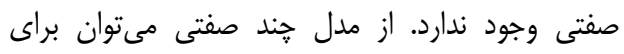

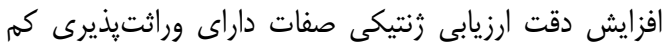

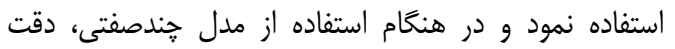

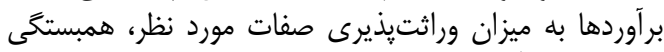

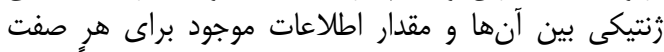

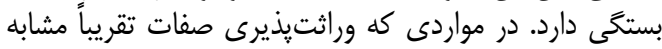

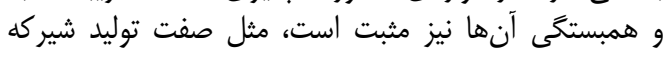

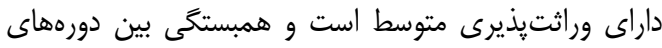

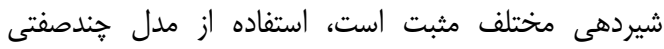

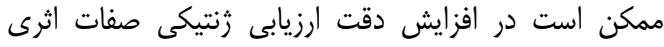

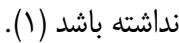

ف $\sigma_{p i j} \quad \sigma_{e i j} \sigma_{a i j}$ افزايشى، محيطى و كوواريانس فنوتيبى،

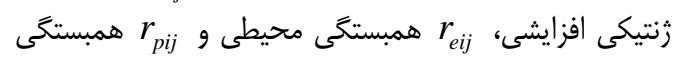

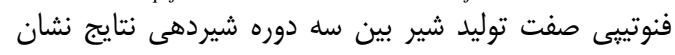

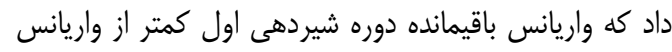

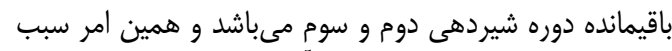

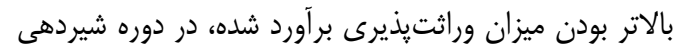

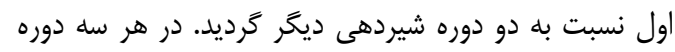

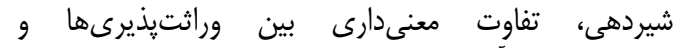
واريانسهاى برآورد شده حاصل از تجزيه تكى صفتى و دو

جدول ه- مؤلفه هاى واريانس و وراثتيذيرى صفت توليد شير سه دوره اول بر اساس تجزيه و تحليل دو صفتى Table 5.Variance components and heritability for milk production in first three lactation based on two-trait analysis

\begin{tabular}{|c|c|c|c|c|c|}
\hline$h_{(\mathrm{SE})}^{2}$ & $\sigma_{p}^{2}$ & $\sigma_{e}^{2}$ & $\sigma_{a}^{2}$ & تجزيه & صفت \\
\hline 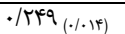 & 11बrब१9 & NEDFYT & TMISV & ميانگَين دوصفتى & \multirow{2}{*}{ شيردهى اول } \\
\hline.$/\left.T \Delta\right|_{(. / .}(T)$ & Marget & Nefiet & rNQT.r & تك صفتى & \\
\hline$\cdot / r \cdot V_{(\cdot / \cdot / r)}$ & IETYq. & ITAVGIF & TrQTAQ & ميانكين دوصفتى & \multirow[b]{2}{*}{ شيردهى دوم } \\
\hline$\cdot / t \cdot f_{(. / . I T)}$ & IgIFIVT & ITADIV. & rrq..r & "ك صفتى & \\
\hline$\cdot / 1 \Delta \cdot(\cdot / \cdot 11)$ & IMAYIIT & $19 \cdot \cdot 141$ & TATQVE & ميانكين دوصفتى & \multirow{2}{*}{ شيردهى سوم } \\
\hline.$/ N F V_{(. / .19)}$ & MVYISV & lQQVET. & TVQVTr & تك صفتى & \\
\hline
\end{tabular}


نتايج نشان داد كه همبستخى زنتيكى و فنوتيبيى بين

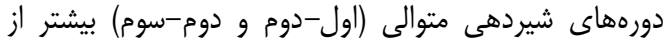

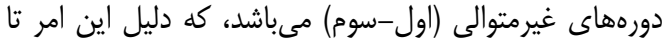

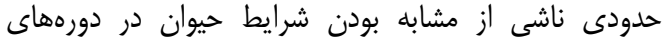

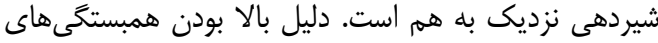

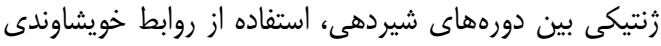

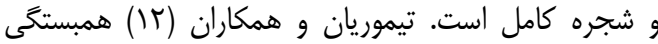

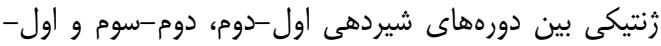

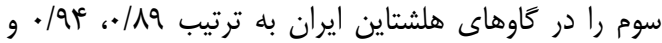

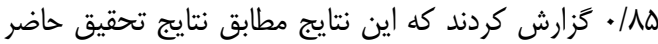

مىباشد.

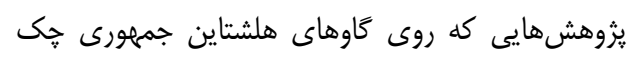

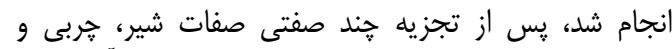

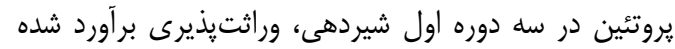

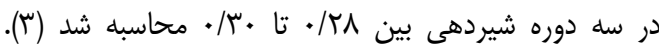

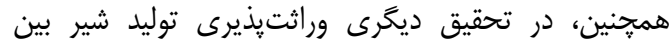

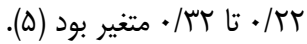

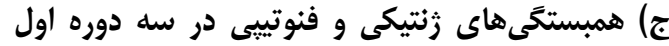

شير دهى

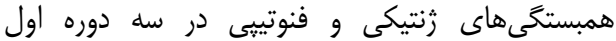

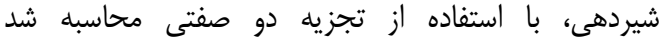

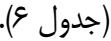

Table 6. Genetic and phenotypic correlation in first three lactation

جدول צ- همبستخى هاى زنتيكى و فنوتيبى در سه شيردهى اول، دوم و سوم

$\frac{1 \Delta \omega}{\cdot / 8}$

$\cdot . / 8 \pi$

.$/ 10$

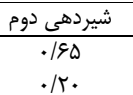

.$/ 91$

شيردهى دوم اول

.$/ 91$

شيردهى سوم دوم

توارث صفات را بررسى كرد، همجنين اطالاع از خارامترهاى

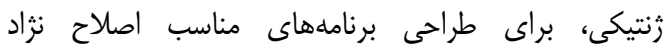

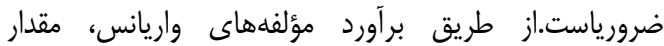

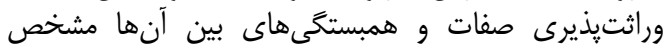

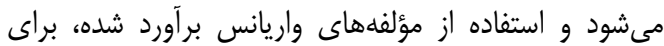

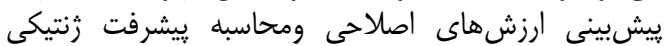
حاصل از آنها ضرورى مى بـاشد.

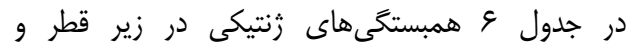

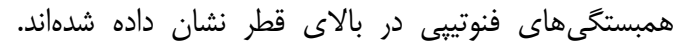

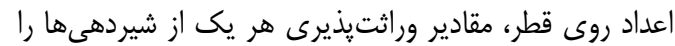
نشان مى دهد. برآورد مؤلفههاى واريانس در اصلار إلاح دام داراى اهميت

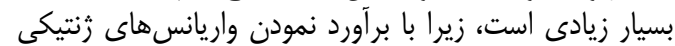

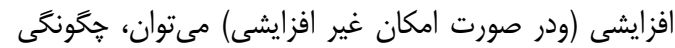

1. Abdolmohammadi, A. 2003. Estimation of genetic parameters for length of productive life and correlation with production traits in Iranian Holstein cattle. M.Sc. thesis, Tehran University, Karaj, Iran. $98 \mathrm{pp}$ (In Persian).

2. Dahlin, A., U.N. Khan, A.H. Zafar, M. Saleem, M.A. Chaudhry and J. Philipsson. 1998. Genetic and environmental causes of variation in milk production traits of Sahiwal cattle in Pakistan. Journal of Animal Science, 66: 307-318.

3. Dedkova, L. and J. Wolf. 2001. Estimation of genetic parameters for milk production traits in Czech dairy cattle populations. Czech Journal of Animal Science, 46: 298-307.

4. Dekkers, J.C.M., P.V. Birke and J.P. Gibson. 1995. Optimum linear selection indexes for multiple generation objectives with non-linear profit function. Journal of Animal Science, 61: 165-175.

5. Garcia- Cortes, L.A., C. Moreno, L. Varona, M. Rico and J. Altarriba. 1995. Co-variance component estimation of yield traits between different using an animal model. Journal of Livestock Production Science, 43: 111-117.

6. Jahandar, M.H. 2002. Estimation of genetic and environmental trend for some production traits in Holstein cattle of Iran. M.Sc. thesis, Tehran University, Karaj, Iran. 135 (In Persian).

7. Kominakis, A., M. Volanis and M. Rogdakis. 2001. Genetic modeling of test day records in dairy sheep in using orthogonal Legendre polynomials. Small Ruminant Research, 39: 209-217.

8. Motamedi, M.M., F. Eftekhar Shahroodi, R. Valizadeh, H. Farhangfar, M. Bashtani and H. Naeemipoor. 2011. Estimation of genetic parameters for some production and reproduction traits in a herd of Holstein cow. Journal of Animal Science (Pajouhesh and Sazandegi), 90: 22-27 (In Persian).

9. Razavi, M., M. Vatankhah, H.R. Mirzaee and M. Rokuee. 2007. Estimation of genetic trends for production traits of Holstein cattle in Markazi province. Journal of Pajouhesh and Sazandegi, 77: 5562.

10. Rostami-Angasi, M. and A. Soudagar-Amiri. 2009. Estimation of genetic parameters for production trait in Holstein dairy cattle in Sari. Journal of Animal Science, 3: 35- 41.

11. Safi Jahanshahi, A., R. Vaez Torshizi, N. Emam Jomae Kashan and M.B. Sayad Nejad. 2003. Estimation of genetic parameters for milk production traits in Iranian Holstein dairy cattle with different animal models. Iranian Journal of Agriculture Science, 34: 177-186 (In Persian).

12. Teimoorian, M., A.A. Aslami Nejad and M. Tahmoores Poor. 2011. Estimation of genetic parameters of production traits in Holstein cattle. Iranian Journal of Animal Science Research, 3: 179-184.

13. Wang, T., R.L. Fernando and S.D. Kachman. 2001. MATVEC User's guide. Version 1.03., Department of Biometry, University of Nebraska: Lincoln, USA. 


\title{
Estimation of Variance Components for Milk Production Traits in Iranian Holstein Cows
}

\begin{tabular}{cc} 
Sheida Varkoohi \\
\hline \hline Assistant Professor, Razi University of Kermanshah \\
(Corresponding author: S.Varkoohi@ gmail.com) \\
Received: October 4, 2014 & Accepted February 4, 2015 \\
\hline \hline
\end{tabular}

\begin{abstract}
In this research, estimation of genetic parameters for milk production trait was performed by using collected data from 1991 to 2010 in Iranian Holstein dairy cattle. Genetic parameters were estimated by MATVEC Microsoft, using animal model, with univariate and multivariate analysis. The data set were including 161328, 123369 and 81013 records for first, second and third lactations, respectively. Based on univariate analysis, heritability estimates for three lactations were $0.25,0.20$ and 0.15 , respectively. Multivariate analysis was performed twice for each lactation and variances and heritability estimates were averaged. Heritability estimates of milk yield were $0.25,0.21$ and 0.15 in first, second and third lactation, respectively, with multivariate analysis. Genetic correlation were $0.95,0.98$ and 0.91 between first-second, second-third and first-third lactations and phenotypic correlations between them were $0.65,0.62$ and 0.55 , respectively. Results showed that the heritability estimates of milk yield were decreased from the first to the third lactation; also, genetic and phenotypic correlations between consecutive lactations (first-second and second-third) were more than inconsecutive lactations (first-third) lactations.
\end{abstract}

Keyword: Genetic parameters, Lactation, Multivariate analysis, Univariate analysis 